\title{
Young Binaries as a Test for Pre-Main Sequence Evolutionary Tracks
}

\author{
Hans Zinnecker \\ Astrophysikalisches Institut Potsdam, An der Sternwarte 16, D-14482 Potsdam, Germany \\ email: hzinnecker@aip.de
}

\begin{abstract}
Observations of young low-mass binaries ( $\mathrm{t} \lesssim 10^{7} \mathrm{yr}, \mathrm{M} \lesssim 3 \mathrm{M}_{\odot}$ ) can be used to calibrate pre-Main Sequence (pre-MS) evolutionary tracks. Recent high angular resolution HST/FGS, speckle, and long-baseline interferometry have resolved the astrometric orbits of a few SB2 pre-MS binaries and have provided the individual dynamical masses of their components as well as the system orbital parallaxes. Spectroscopic fits and filter photometry have permitted to determine SpT (temperatures) and a good estimate of the absolute magnitude (bolometric luminosity) of the components, which in turn allows one to place the components on a theoretical HR-diagram. In this way, one can check (a) whether the measured dynamical masses agree with the predicted masses on the tracks and (b) whether both components lie on an isochrone, as they should for a coeval physical pair of stars.

With a sufficiently large sample of different masses and ages of resolved SB2 systems, most of the parameter space of pre-MS tracks can be tested, even for very low stellar masses $\left(\mathrm{M}<0.5 \mathrm{M}_{\odot}\right)$ and very young ages $(<2 \mathrm{Myr})$. This is a prerequisite in order to derive the IMF and star formation history in very young clusters and associations.
\end{abstract}

Keywords. techniques: high angular resolution, techniques: interferometric, techniques: spectroscopic telescopes, astrometry, binaries: spectroscopic, binaries: visual, stars: fundamental parameters, Hertzsprung-Russell diagram, stars: late-type, stars: pre-main-sequence

\section{Introduction}

I would like to begin my contribution by paying tribute to Czech born Prof. Zdeněk Kopal, one of the fathers of close binary star astrophysics, who died in 1993 and is buried around the corner in the Prague Vyšehrad cemetery. I met him during the total solar eclipse in June 1983 in Indonesia (cf. Kopal 1986) following IAU Coll. No. 80, of which he is the co-editor. It was he (and the late Dr. Rahe from Bamberg) who invited me to present a first review on "binary statistics and star formation" (Zinnecker 1984). This was also my first major paper for an IAU-related event, and I am still proud of it, although to this day hardly anyone took note of it (except T. Oswalt in his talk yesterday). Indeed, this paper now provides an interesting look back in time to what we knew in $\sim 1980$ about binary frequency and multiplicity, mass ratio and period distribution. Those were the days when star formation started to take off as a subject, and an early confrontation between observations and theory became possible.

Ten years later, an excellent ARAA review on pre-MS binaries was published (Mathieu 1994). Then, in the year 2000, I hope some of you remember, IAU-Symp. 200 "The Formation of Binary Stars" was held in Potsdam (eds. Zinnecker \& Mathieu 2001).

In today's meeting the topic that I want to address is not so much the formation of binary stars but more the use of young pre-MS binaries for checking that we have reliable masses and ages for low-mass pre-MS stars and brown dwarfs. This context already played a role in Mathieu's (1994) review, but in the meantime more and better data are becoming available, mainly through the advent of long-baseline interferometry. A rationale for 
interferometry and a summary of results on dynamical mass determinations are given in Zinnecker \& Correia (2004) and Hillenbrand \& White (2004), respectively. Here I will try to explain the logic behind the use of young binaries for testing early stellar evolution. In addition, the latest results on dynamical mass measurement of young stellar objects will be presented. A more comprehensive review of this topic is the article by Mathieu et al. (2006) in the proceedings of Protostars and Planets $V$ (highly recommended to read).

\section{Pre-MS tracks}

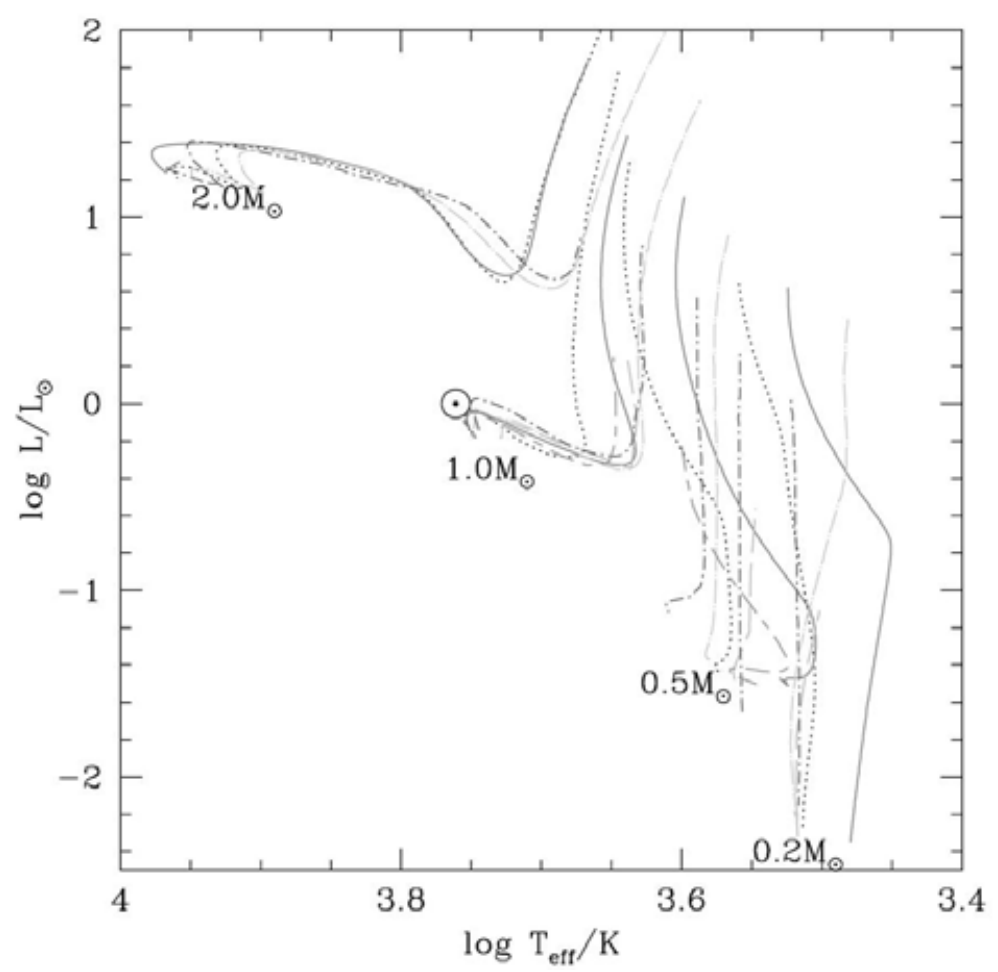

Figure 1. Variation between pre-main-sequence contraction tracks for masses $0.2,0.5,1.0$, and $2.0 \mathrm{M}_{\odot}$; for the following models: S93-Siess (solid line), DM97-d'Antona \& Mazzitelli, with 1998 correction (dotted line), B98, $\alpha=1.9$-Baraffe et al. (long-dashed line), PS99-Palla \& Stahler (dot-short-dashed line), S00-Siess (dot-long-dashed line), and $\mathrm{Y}^{2}-$ Yi/Yale (longdash-short-dashed line). Note that the PS99 models, for which no $0.5 \mathrm{M}_{\odot}$ track is available, have both the 0.4 and the $0.6 \mathrm{M}_{\odot}$ tracks plotted instead. Note also that the $\mathrm{Y}^{2}$ models do not extend as low as $0.2 \mathrm{M}_{\odot}$ (figure \& caption from Hillenbrand \& White 2004).

Figure 1 shows pre-MS tracks for $0.2,0.5,1.0$, and $2.0 \mathrm{M}_{\odot}$ as calculated by various authors (given in the figure caption). The near vertical parts of the curves correspond to the so-called Hayashi tracks (fully convective quasi-statically contracting stars), while the more horizontal parts leading on to the Main Sequence correspond to a stellar structure where the energy transport in the interior is mainly radiative (so-called Henyey tracks). A good description of pre-MS evolution and a reference set of pre-MS evolutionary tracks with isomasses and isochrones can be found in the new textbook on 'The Formation of Stars' by Stahler \& Palla (2004; see their Figure 1.18).

One can easily see from Figure 1 that the pre-MS models differ quite substantially for low masses, particularly below $0.5 \mathrm{M}_{\odot}$ where molecular opacities start playing a role in 
the cool atmospheres. It can happen that someone's $0.2 \mathrm{M}_{\odot}$ star is another one's $0.5 \mathrm{M}_{\odot}$ star, for a given point in the bottom right corner of the HR-diagram. Such a big error is clearly unacceptable, and the model input must be fixed or calibrated by appropriate observations.

Table 1. Important young spectroscopic binaries for dynamical mass determinations

\begin{tabular}{lll}
\hline References & Objects & Type \\
\hline Covino et al. 2000, A\&JA Lett. & RXJ 0529.4+0041 & SB2E \\
Steffen et al. 2001, AJ & $045251+3016$ & SB2A \\
Boden et al. 2005, ApJ & HD 98800B & SB2A \\
Schaefer, Simon, Prato 2006, ESO conf. & Haro 1-14c & SB2A \\
Stassun, Mathieu, Valenti 2006, Nature & 2MASS 0535-05 & SB2E \\
\hline
\end{tabular}

\section{Logical steps for testing pre-MS tracks}

The first step is, of course, to calculate a set of pre-MS tracks on the computer, using certain input physics (e.g., convection described by a mixing-length parameter or otherwise, opacity sources, metallicity, or even magnetic star spots). Different assumptions on the main input physics lead to substantially different evolutionary tracks.

The next step then is to select those tracks that best describe the early evolution of real stars rather than model stars. How to do this? The trick is to use young low-mass spectroscopic binaries in a variety of star-forming regions (young clusters and associations) with measured orbital periods (and inferred component separations) such that they can be spatially resolved and their orbits followed by high-angular resolution interferometric observations. The relevant periods are around $1 \mathrm{yr}$, give or take a factor of $3-5$ (i.e., semi-major axes of the order of $1 \mathrm{AU}$ within a factor of $2-3$ ), depending on the actual distance $(50 \mathrm{pc}-150 \mathrm{pc})$. Young eclipsing binaries would be even better but they are very rare and only very few have been found (see the results section). Note that a semi-major axis of $1 \mathrm{AU}$ at $50 \mathrm{pc}$ (e.g., TW Hydrae association) or $150 \mathrm{pc}$ (e.g., the Sco Cen association) correspond to angular separations of 20 mas and 7 mas, respectively, fairly easy to do with Keck or VLTI measurements in the near-IR $J H K$ bands. Because long-baseline interferometers such as Keck and VLTI have only recently begun to operate, it is no wonder that only very few examples have been observed.

In more detail, the useful young binary objects have to meet two more requirements:

a) they had better be weak-line or naked T Tauri stars rather than classical T-Tauri stars. The former have the advantage of possessing no circumstellar or circumbinary accretion disks, hence their bolometric luminosity need not be corrected for accretion luminosity (which in turn requires observing their spectral energy distribution and an appropriate subtraction). The only correction required is for foreground extinction and reddening.

b) they had better be SB2 systems rather than SB1s. Only the combination of SB2 radial velocity curves and a resolved astrometric orbit yields the orbital inclination (sin i), thus giving the individual masses of the components and, importantly too, the so-called orbital parallax. In case we have an SB1, all is not lost, as there is every chance now to transform an SB1 into an SB2 using near-IR rather than optical spectroscopy (Mazeh et al. 2003, Prato et al. 2003). The reason is that the faint secondary component tends to be cooler and hence relatively brighter w.r.t. the primary in the near-IR, hence the brightness is more nearly equal to unity in the near-IR than in the optical.

The third step is to place the resolved binary components into a theoretical pre-MS HR-diagram or color-magnitude diagram. This is not easy, as we need to get separate SpT 
and stellar luminosities for the components (being careful to avoid hidden triple systems). How to achieve this? Stellar luminosities, particularly for the fainter secondary, can be estimated from the total luminosity of the system and the brightness ratio in at least one filter; the more filters, the better (note here that the VLTI/AMBER instrument provides measurements in all the $J H K$ filters simultaneously). With more than one filter, we also obtain useful component color information. The decomposition of the spectral types is done in the near-IR, using true near-IR spectral template stars which were measured before and tabulated. Then two spectra are superimposed, starting from an initial guess, and iterated, until the observed spectrum of the binary system is matched (in a chi-square sense). A list of interesting young spectroscopic binaries (SB's) is given in Table 1.

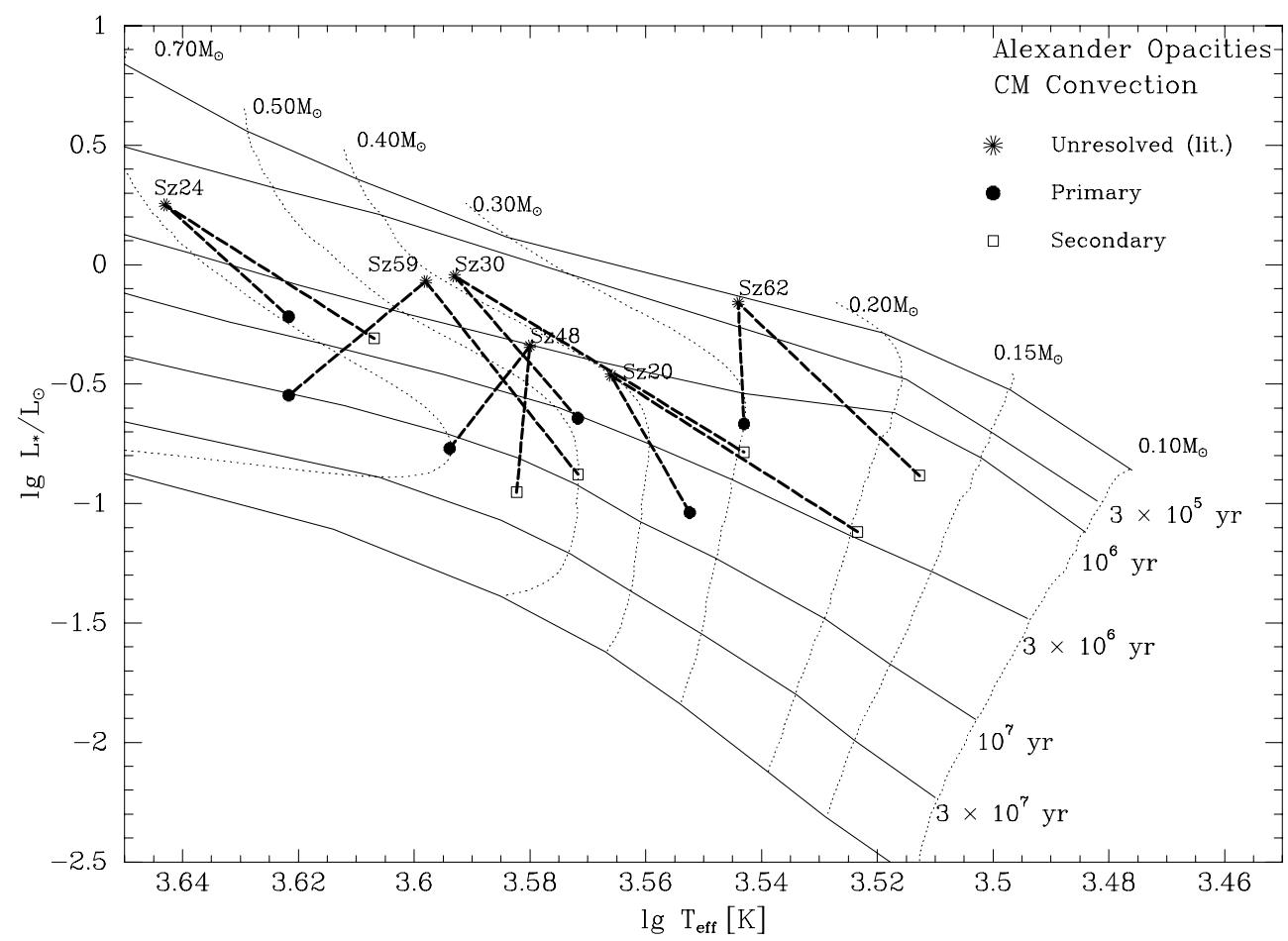

Figure 2. Comparison of literature values for the unresolved binaries with results for the individual binary components (from Brandner \& Zinnecker 1997, using D’Antona \& Mazzitelli 1994 tracks). Unresolved binaries lead to an underestimate of the age of a T Tauri star population.

As an aside, it is interesting to check, in a kind of a thought experiment, how wrong one can go in terms of pre-MS masses and ages when the binary nature of a young spectroscopic or close visual binary system is unrecognized as opposed to be recognized and resolved (see Figure 2). One can see that pre-MS masses and ages can change by more than $100 \%$ (e.g., Sz 48, Sz 59 in Figure 2). Indeed, overestimating the stellar luminosity in an unresolved pre-MS binary whose components are on Hayashi tracks produces an underestimate of the system age, as already noticed by Simon, Ghez, \& Leinert (1993). As a consequence, as these authors have pointed out, the average age of a young group of stars (such as the T Tauri stars in Taurus, whose binary frequency is close to $100 \%$ ) is $2-3$ times longer than it would be if all its binaries were regarded as unresolved objects! 


\section{Results}

\subsection{Previous results}

It was at the IAU Symposium 200 in Potsdam six years ago that the first significant number of dynamical mass measurements of pre-Main Sequence stars were reported, although the very first measurement (the pre-MS secondary of the SB1 eclipsing binary system EK Cep) goes back to Popper (1987). At IAU-S200, M. Simon gave a review on dynamical masses of young stars that focussed primarily on Keplerian rotation measurements of spatially resolved circumstellar disks around single pre-MS T Tauri stars (his own work in collaboration with Dutrey \& Guilloteau at IRAM/PdBI using ${ }^{12} \mathrm{CO} \mathrm{J}=2-1$ maps). Masses of the following six stars were reported: MWC 480, LkCa 15, DL Tau, GM Ori, DM Tau, and CY Tau, with values ranging from $1.65 \mathrm{M}_{\odot}$ down to $0.55 \mathrm{M}_{\odot}$. Errors on the masses were $3-15 \%$, except for the latter where the error exceeds $50 \%$. These errors do not include the imprecision of the distance to the sources, which may add an extra $10-20 \%$. [BP Tau's mass was also listed, but later an improved value was published (Dutrey et al. 2003). Also, UZ Tau E is a spectroscopic binary with a circumbinary disk, so that the binary system mass could be determined (Prato et al. 2002).]

At the same IAU meeting, E. Covino et al. announced the first pre-MS eclipsing binary among double-lined T-Tauri stars in Orion (RXJ 0529.4+0041) and gave preliminary masses $\left(\mathrm{M}_{1}=1.25 \mathrm{M}_{\odot}, \mathrm{M}_{2}=0.91 \mathrm{M}_{\odot}\right)$, while L.P. Vaz discussed the TY CrA system, a Herbig Ae/Be star and hierarchical triple with an eclipsing pair and wider tertiary star. The component masses of the eclipsing pair are $3.16 \mathrm{M}_{\odot}$ (primary, near the ZAMS) and $1.64 \mathrm{M}_{\odot}$ (secondary, pre-MS, estimated age $3 \mathrm{Myr}$ ). The mass of the third member of the system is unknown, its orbit may be highly inclined w.r.t. the inner pair (Casey et al. 1998).

Furthermore, R. White described how to use the hierarchical quadruple system GG Tau (consisting of two close visual pairs, about $10^{\prime \prime}$ apart, with individual component separations of $0^{\prime \prime} .25$ and $1^{\prime \prime} .45$, respectively) to test pre-MS evolutionary models, especially under the assumption of coeval formation. Higher order multiple systems are in principle more powerful test objects than pure binary systems, if all components (including brown dwarfs) can be placed on the HR-diagram.

Last but not least, F. Palla confronted pre-MS models with observations of four intermediate-mass pre-MS spectroscopic eclipsing binaries (1 SB2: RS Cha; 3 SB1s: EK Cep, BM Ori, TY CrA) and found rather good agreement with his own models (Palla \& Stahler 1999). (We omit here the non-eclipsing young SB2s: V773 Tau, NTTS 162814, and P1540, for which only mass ratios but no individual masses can be determined).

Taking all the above information from IAU-S200 together, there have been more than a dozen dynamical masses of young stellar objects gathered up to the year 2000, but the constraints on pre-MS evolutionary tracks from them were not very strong mainly because of a lack of accuracy.

\subsection{Recent results}

A new era of dynamical mass measurements for pre-MS stars began with the publication of the first astrometric-spectroscopic orbit of NTTS 045251+3016 by Steffen et al. 2001 (already presented as a poster at IAU-S200). They used a double-hybrid technique: 1) they patiently resolved a good part of the $7 \mathrm{yr}$ SB1 orbit with the HST/FGS and 2) they transformed the optical light SB1 into an SB2 by near-IR spectroscopy. They thus could derive the inclination angle of the orbit, hence the individual masses, and indeed also the orbital parallax, as discussed in Section 3. The measurements of the primary and secondary component masses $\left(1.45 \mathrm{M}_{\odot}\right.$ and $\left.0.81 \mathrm{M}_{\odot}\right)$ were precise enough 
$(\sim 10 \%)$ to indicate that the Baraffe et al. (1998) tracks (with mixing-length parameter $\alpha=1.0$ ) provided the best fit to the observations. These tracks then predict an age of the system.
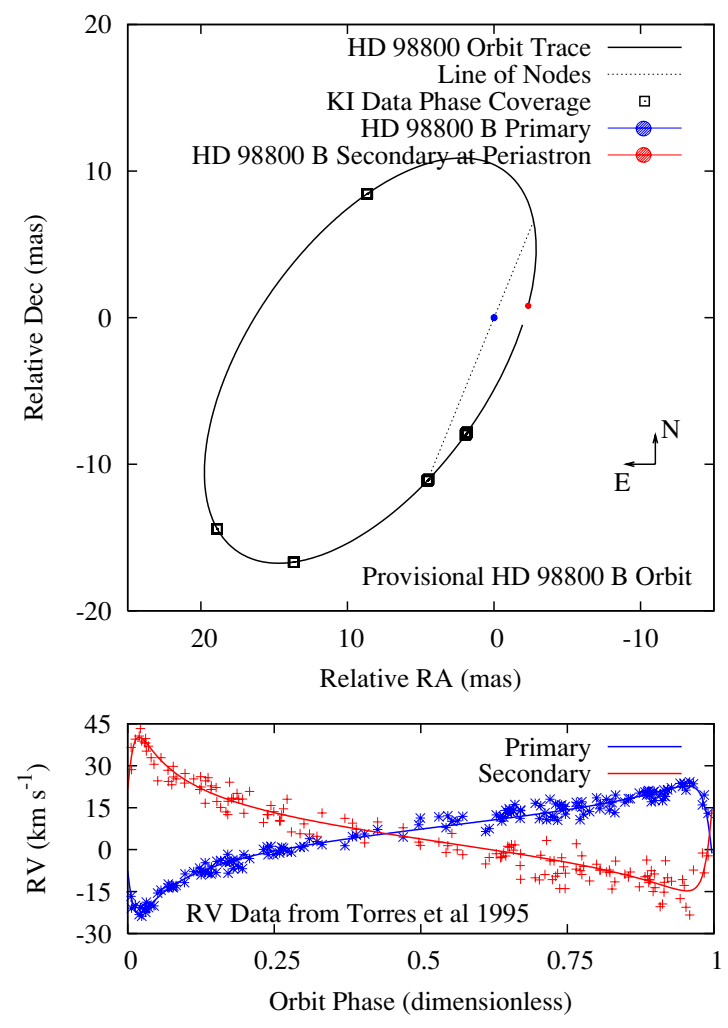

Figure 3. Astrometric-spectroscopic orbit of HD 98800 B (Boden et al. 2005).

It took several years until this feat was sort of repeated for another system: HD $98800 \mathrm{~B}$ in the very nearby TW Hya association (Boden et al. 2005). These authors combined FGS data with ground-based $K$-band Keck interferometric visibility data to derive an astrometric orbital solution for this one binary in a pre-MS quadruple system. Note that the visibility data were compared directly to model predictions in order to constrain the astrometric orbit, without an intermediate determination of the separation. Boden et al. (2005) find that the component masses $\left(\mathrm{M}_{1}=0.70 \pm 0.06 \mathrm{M}_{\odot}\right.$, $\left.\mathrm{M}_{2}=0.58 \pm 0.05 \mathrm{M}_{\odot}\right)$, luminosities and effective temperatures of HD 98800B are inconsistent with solar-metallicity evolutionary tracks; they suggest that a lower metal abundance by a factor of $2-3$ would resolve the discrepancy. The derived orbital parallax of the system is $\sim 42 \mathrm{pc}$.

This forefront observational study bodes well for a significant number of masses being measured via interferometry and spectroscopy in the near future. Indeed, Haro 1-14c has also been studied using the Keck interferometer (Schaefer, Simon, \& Prato 2006). The results indicate that the secondary is likely to have a mass of about $0.4 \mathrm{M}_{\odot}$, the lowestmass pre-MS tested to date (excluding brown dwarfs). Similarly low-mass secondary components may prove most valuable in the future, because pre-MS models are least constrained in the lowest-mass regime (see Section 2). 
Another highlight was the discovery of the eclipsing binary young brown dwarf 2MASS J05352184-0546085 in the Orion Nebula star formation region (Stassun, Mathieu, \& Valenti 2006) with component masses of about 54 and 34 Jupiter masses and radii of about 0.67 and $0.51 \mathrm{R}_{\odot}$ (obtained from a combined analysis of the light curve and orbit solution). Radial velocities of the two components were obtained at eight distinct epochs with the Phoenix high-resolution near-infrared spectrograph at the Gemini South telescope. The orbital period is about 10 days. Surprisingly the less-massive brown dwarf is the hotter of the pair, a fact for which no reasonable explanation has been found up to now (but see Wuchterl \& Tscharnuter 2003).

Stassun et al. (2004) had earlier discovered a pre-MS stellar eclipsing binary (V1174 Ori with $\mathrm{M}_{1}=1.01 \mathrm{M}_{\odot}$ and $\left.\mathrm{M}_{2}=0.73 \mathrm{M}_{\odot}\right)$. Eclipsing pre-MS binaries offer of course an extra advantage over astrometric-spectroscopic binaries: a measurement of stellar radii which are also a predicted outcome from pre-MS evolutionary models and hence help to further confront theory with observations (for details see Mathieu et al. 2006).

Duchêne et al. (2006) recently published stellar masses in the T Tau triple system, especially those of $\mathrm{T}$ Tau Sa and T Tau Sb $\left(2.73 \pm 0.31\right.$ and $0.61 \pm 0.17 \mathrm{M}_{\odot}$, respectively). Taking advantage of $\mathrm{T}$ Tau $\mathrm{N}$ as a reference, absolute astrometric monitoring of the Keplerian motion of the $\mathrm{T}$ Tau $\mathrm{S}$ binary system was possible, using $K$-band infrared speckle interferometry at the Keck telescope to fit the orbit.

Further, a $240 \mathrm{AU}$ wide planetary mass young binary (Oph 1622), with model masses of 14 and $7 \mathrm{M}$ (Jupiter), has been identified with the VLT (Jayawardhana \& Ivanov 2006).

Finally, another exciting result became known during the present IAU Symposium 240: the star $\theta^{1}$ Ori E, a member of the Orion Trapezium cluster, has been detected as a double-lined spectroscopic binary (Herbig \& Griffin 2006) with estimated masses of 3 $4 \mathrm{M}_{\odot}$ and a period of 9.89 days (see also Costero 2007). This object is the second brightest X-ray source in the Trapezium cluster after $\theta^{1}$ Ori $\mathrm{C}$, itself resolved as a sub-arcsecond speckle binary (see Schertl et al. 2003 for orbital motion).

\subsection{Summary}

Where does that leave us? Most of the results mentioned above have been discussed in two recent comprehensive reviews: Hillenbrand \& White (2004) and Mathieu et al. (2006, PPV). However, the last 6 years have not been too prolific in providing many new results, and progress has rather stalled, perhaps because the "Golden Age of Astrometry" (quoting M. Simon) is close but hasn't quite arrived yet. Nevertheless VLTI, Keck-I, and CHARA observations of more pre-MS binaries will come in within the next few years. Already a target list of some eight pre-MS spectroscopic binaries has been elaborated for VLTI/AMBER (Guenther et al. 2007, see also Melo et al. 2001), ready to be observed. An amazing spectroscopic triple system is BS Indi in the young Tucana association: it consists of two eclipsing M0V stars orbiting a K0V star with a period of 3.3 years, readily resolvable with the VLTI. Stay tuned!

Yet, now that dynamical mass measurements will become more frequent with the application of ground-based optical/infrared interferometers, the primary limitations to such tests will be systematic errors in determining the stellar properties necessary for the comparison with evolutionary models, in complete agreement with Mathieu et al. (2006).

\section{Acknowledgements}

I thank Drs. S. Correia and E. Guenther for insightful discussions about the most recent results in the literature. I also thank Bob Mathieu and Mike Simon for valuable information during the preparation of this talk. Last but not least I thank the IAU and the NOC chairman of the Prague General Assembly, Jan Palouš, for financial support. 


\section{References}

Baraffe, I., Chabrier, G., Allard, F., \& Hauschildt, P.H. 1995, ApJ 446, L35 (B98)

Boden, A.F. et al. 2005, ApJ 635, 442

Brandner, W. \& Zinnecker, H. 1997, A\&3A 321, 220

Casey, B.W., Mathieu, R.D., Vaz, L.P.R., Andersen, J., \& Suntzeff, N.B. 1998, AJ 115, 1617

Costero, R., Poveda, A., \& Echevarría, J. 2007, these proceedings, 130

Covino, E., Catalano, S., Frasca, A., Marilli, E., Fernández, M., Alcalá, J.M., Melo, C., Paladino, R., Sterzik, M.F., \& Stelzer, B. 2000, A\&SA 361, L49

D'Antona, F. \& Mazzitelli, I. 1994, ApJS 90, 467

D'Antona, F. \& Mazzitelli, I. 1997, Mem. Soc. Astr. It. 68, 807 (DM97)

D'Antona, F., Ventura, P., \& Mazzitelli, I. 2000, ApJ 543, L77

Duchêne, G., Beust H., Adjali F., Konopacky Q.M., \& Ghez, A.M. 2006, A\&A 457, L9

Guenther, E., Esposito M., Mundt R., Covino E., Alcalá, J.M., \& Stecklum B. 2007, astro$\mathrm{ph} / 2268 \mathrm{G}$

Guenther, E.W., Covino, E., Alcalá, J.M., Esposito, M., \& Mundt, R. 2005, A $ళ$ A 433, 629

Herbig, G.H. \& Griffin, R.F. 2006, AJ 132, 1763

Hillenbrand, L.A. \& White, R.J. 2004, ApJ 604, 741

Jayawardhana, R. \& Ivanov, V.D. 2006, Science 313, 1279

Kopal, Z. 1986, Of stars and men. Reminiscences of an astronomer (Bristol: Hilger)

Mathieu, R.D. 1994, ARAESA 32, 465

Mathieu, R., Stassun, K., Baraffe, I., Simon, M., \& White, R. 2006, in: B. Reipurth, D. Jewitt, K. Keil (eds.), Protostars and Planets $V$, in press

Mazeh, T., Simon, M., Prato, L., Markus, B., \& Zucker, S. 2003, ApJ 599, 1344

Melo, C.H.F., Covino, E., Alcalá, J.M., \& Torres, G. 2001, A $\mathscr{E} A$ 378, 898

Palla, F. \& Stahler, S.W. 1999, ApJ 525, 772 (PS99)

Popper, D.M. 1987, ApJ 313, L81

Prato, L., Greene, T.P., \& Simon, M. 2003, ApJ 584, 853

Prato, L., Simon, M., Mazeh, T., Zucker, S., \& McLean, I.S. 2002, ApJ 579, L99

Schaefer, G.H., Simon, M., \& Prato, L. 2006, in: A. Richichi, F. Paresce (eds.), The Power of Optical/IR Interferometry, in press

Schertl, D., Balega, Y.Y., Preibisch, Th., \& Weigelt, G. 2003, A $\& A$ 402, 267

Siess, L., Dufour, E., \& Forestini, M. 2000, A $6 A$ 358, 593 (S00)

Simon, M., Ghez, A.M., \& Leinert, Ch. 1993, ApJ 408, L33

Stahler, S.W. \& Palla, F. 2004, The Formation of Stars (Wiley-VCH)

Stassun, K.G., Mathieu, R.D., \& Valenti, J.A. 2006, Nature 440, 311

Stassun, K.G., Mathieu, R.D., Vaz, L.P.R., Stroud, N., \& Vrba, F.J. 2004, ApJS 151, 357

Steffen, A.T., Mathieu, R.D., Lattanzi, M.G., Latham, D.W., Mazeh, T., Prato, L., Simon, M., Zinnecker, H., \& Loreggia, D. 2001, $A J$ 122, 997

Wuchterl, G. \& Tscharnuter, W.M. 2003, A\&A 398, 1081

Yi, S.K., Kim, Y.-C., \& Demarque, P. 2003, ApJS 144, 259 (Yi/Yale)

Zinnecker, H. 1984, ApESSS 99, 41

Zinnecker H. \& Correia, S. 2004, in: R.W. Hilditch, H. Hensberge, K. Pavlovski (eds.), Spectroscopically and Spatially Resolving the Components of the Close Binary Stars, ASP Conference Series, Vol. 318 (San Francisco: Astronomical Society of the Pacific), p. 34

\section{Discussion}

G. Wuchterl: You showed the Boden et al. results that show an offset of the Siess tracks relative to the observations. Collapse models (Wuchterl \& Tscharnuter 2003) predict such a shift to be a few hundred Kelvin $\left(500 \mathrm{~K}\right.$ at $1 \mathrm{M}_{\odot}$ and $\left.\sim 2 \mathrm{~L}_{\odot}\right)$. How large is the shift in temperature that you discussed?

ZINNECKER: Let's have a look at Figure 6 in Boden et al. (2005). One can see that the temperature offset is $\sim 200 \mathrm{~K}$ for solar metallicity models, both for the primary $\left(\sim 0.7 \mathrm{M}_{\odot}\right)$ and for the secondary $\left(\sim 0.6 \mathrm{M}_{\odot}\right)$, in the sense that the observed temperatures are hotter than those consistent with the dynamical iso-masses. 